\title{
Sobre la relación entre el conocimiento y el pensamiento simbólico: algunos aportes fundamentales para las ciencias sociales
}

\section{About the relationship between knowledge and the symbolic thought: some fundamental contributions for the social sciences}

Juan Erick Carrera (patriciocarrera.a@gmail.com) Facultad de Ciencias Sociales y Humanidades, Universidad Católica de Temuco (Temuco, Chile) ORCID: 0000-0003-0026-6565

\begin{abstract}
This paper proposes a particular form of problematize what we can understand as knowledge in social sciences, that marginalized from a naturalistic perspective and closer to a phenomenologicalhermeneutics glimpses possible intersections between the symbolic, the social and the cognitive, with the purpose of establishing from a complex and dialectical dimension an analytical approach of the social science of knowledge. To do this, we will begin by figuring out a problematization about knowledge in social sciences, addressing some sociological premises and socio-cognitive functions to conceptualize a complex perspective of knowledge. Subsequently, a second section is based on three main axes: objectivity, subjectivity and imagination, as a transcendental triad in this epistemological perspective. To end with the search for the relationship between knowledge and the symbolic thought according to the arguments outlined in the preceding paragraphs, where we understood that the imaginary-archetypal structures surrounding the social reality, the process of understanding and intersubjectivity constitute, among others, the foundations of which we will understand by knowledge in social sciences
\end{abstract}

Key words: social knowledge, objectivity, subjectivity, symbolic thought, social sciences.

\section{Resumen}

En el presente artículo se propone una forma particular de problematizar lo que podemos comprender como conocimiento en ciencias sociales, que marginado de una perspectiva naturalista y más próximo a una fenomenológico-hermenéutica, vislumbra posibles intersecciones entre lo simbólico, lo social y lo cognitivo, con la finalidad de establecer desde una dimensión compleja y dialéctica un enfoque analítico de la ciencia social del conocimiento. Para ello, comenzaremos por figurar una problematización sobre el conocimiento en ciencias sociales, abordando algunas premisas sociológicas y las funciones sociocognitivas para conceptualizar una perspectiva compleja del conocimiento. Posteriormente, un segundo apartado se sustenta sobre tres ejes principales: la objetividad, la subjetividad y la imaginación, como una tríada trascendental en esta perspectiva epistemológica. Para finalizar con la búsqueda de la relación entre el conocimiento y un pensamiento simbólico de acuerdo con los argumentos expuestos en los apartados anteriores, donde se entiende que las estructuras imaginario-arquetípicas circundantes en 
la realidad social, el proceso de comprensión e intersubjetividad constituyen, entre otros, los cimientos de los que vamos a entender por conocimiento en ciencias sociales.

Palabras clave: conocimiento social, objetividad, subjetividad, pensamiento simbólico, ciencias sociales.

\section{Introducción}

El problema del conocimiento en ciencias sociales y sobre todo en filosofía es de larga data, pero quizá sea el texto La estructura de las revoluciones científicas de Thomas Kuhn, publicado originalmente en 1962, el cual marque un precedente respecto a la situación histórica de los postulados de la ciencia y sus crisis paradigmáticas, donde los relativismos expresan ideas de ruptura y discontinuidades inherentes a los contextos históricos y, en consecuencia, sustantivos en el ejercicio de un trabajo científico que adquiere diversas identidades, pues: "el papel determinante del contexto socio-histórico-cultural en el que se producen los 'descubrimientos' constituye el eje de una auténtica revolución epistemológica que afecta gravemente al concepto mismo de verdad" (Vidal 2011:23). Por lo que la realidad debe visualizarse desde una búsqueda intencionada del actuar en y para el mundo, en tanto la forma de observar analíticamente los fenómenos sociales obedece siempre a modelos de conocimiento que funcionan como una guía en la investigación y, por consecuencia, le otorgan un sentido teórico. Estos esquemas "representan una visión histórica singular del mundo, en la medida en que se asientan en principios metafísicos generales, los paradigmas actúan como herramientas cognitivas que abarcan todo lo necesario para el desenvolvimiento de la labor investigadora en lo concerniente a la metodología, los presupuestos teóricos, los modelos de aplicación sistemática de las mismas, etc." (Vidal 2011:24). Lo sustancial de esto es el proceso de ruptura histórica entre diferentes esquemas operantes en las bases del conocimiento científico, donde el giro cognoscitivo desencadenará el proceso de crisis y establecerá nuevos paradigmas, encaminando el cambio científico a nuevos tiempos.

No obstante, la obra de Kuhn ataca más profundamente, situando al pensamiento científico como un elemento crucial en la disolución o mantención de estos paradigmas mediante ciertas predisposiciones mentales, por ejemplo las dimensiones divergente y convergente; por la primera se entiende esa predisposición científica a una ruptura con directrices pretéritas, proliferando así una perspectiva epistemológica de cambio; mientras que la segunda se sostendría por consensos establecidos, provocando una inherente fricción entre ambas: "de modo tal que no existan dos de esas articulaciones completamente iguales, con un éxito parcial cada una de ellas ni con el suficiente éxito como para poder ser aceptadas como paradigmas por el grupo. A través de esta proliferación de coyunturas divergentes (de manera cada vez más frecuente llegarán a describirse como ajustes ad hoc), las reglas de la ciencia normal se hacen cada vez más confusas" (Kuhn 1971:137). Diremos entonces que: "Los paradigmas ejercen sobre la mente de los científicos una influencia implícita que les hace aceptar algunas cuestiones básicas dándolas por sobreentendidas. Su formación y la tradición de la investigación que comparten proponen la coherencia necesaria para evitar cuestionamientos radicales" (Brunetti 2013:198).

Asimismo, los procesos de conocimiento influenciados por estos cambiantes esquemas históricos no carecen de identidad propia, debido a las dimensiones simbólicas que ejercen fuerza sobre el contexto social donde se constituyan, configurando la posibilidad de modelos epistémicos sui generis a partir de los distintos marcos socioculturales situados en un tiempo y espacio determinado. Pues, el mismo proceso de construcción de conocimientos supone la existencia de un soporte intersubjetivo que resulta necesario para producir esquemas de inteligibilidad que hacen posible las relaciones sociales y las explicaciones del mundo, expresados mediante sentidos compartidos en el decurso de la experiencia humana y social. Esta 
dimensión fecunda las posibilidades sociofenomenológicas de investigación, desentendiéndose de ese distanciamiento histórico entre el investigador y su objeto analítico de estudio: "Phenomenology of Intersubjectivity manner of investigation is directed instead to objects of perception, the nature of which is said to exist independently of the subject who conducts the investigation and whose reality is presumed to exist independently of the investigator" (Thomsom 2005:38). A esa estructura simbólica que vincula en primera instancia al individuo con el mundo, y que fundamenta su condición subjetiva, le llamamos pensamiento simbólico y tiene como función alimentar el magma de significados sociales (lebenswelt) que instituyen ideas sobre la realidad, construyendo modelos interpretativos del mundo y otorgando sentidos a la existencia, pues: "Del mundo conocido y de las experiencias intersubjetivas compartidas por los sujetos, se obtienen las señales, las indicaciones para interpretar la diversidad de símbolos" (Rizo 2005:23).

De modo que los diversos conocimientos en ciencias sociales, al confluir desde redes simbólicas particulares, constituyen "una forma de conciencia social que se construye históricamente como un sistema ordenado de conocimientos en forma de conceptos, juicios teóricos, hipótesis teóricas y leyes teóricas" (Narváez 2014:228). Todo esto involucra un proceso complejo, donde el conocimiento es, en algún sentido, un producto elaborado desde la percepción, deviene de ideas que se articulan en virtud de impresiones de la realidad modeladas por aquellas formas simbólicas que dominan un contexto sociocultural y las relaciones humanas, en tanto 'los individuos actuarían con referencia al 'otro' en términos de los símbolos desarrollados mediante la interacción, haciéndolo a través de la comunicación de éstos" (Gadea 2018:41), llevando a que todo el proceso de construcción de conocimientos, aunque gocen de influencias paradigmáticas, siempre se nutra de las estructuras simbólicas y su opus operatum en la vida social.

De esta forma, este artículo pretende construir una triangulación de perspectivas que, en su convergencia, nos otorguen los elementos para dilucidar el problema que presenta acercarnos a una definición del conocimiento social, sin dejar de lado la influencia simbólica que alimenta la experiencia humana. Comenzaremos, en un primer apartado, problematizando el conocimiento desde las ciencias sociales y elaborando un corpus teórico-epistemológico que navegue por una serie de postulados que expresan una idea fundamental: la construcción del conocimiento desde una relación intrínseca entre el individuo y el mundo. Para ello, enunciaremos elementos que provienen desde la filosofía y las ciencias sociales, para comprender que el conocimiento se expresa como un constructo sociocultural que emerge desde las formas simbólicas que se manifiestan en la relación entre diversos actores del mundo social, con la finalidad de comprender e interpretar elementos abstractos para validarlos e instituirlos mediante la interpretación y significaciones sociales. Posteriormente nos centrarnos en el debate más clásico sobre la dicotomía entre el conocimiento científico y el sentido común.

Un segundo apartado trata sobre una tríada fundamental: la relación entre objetividad, subjetividad e imaginación, donde se pretenderá profundizar en los elementos del apartado primero, estableciendo las cualidades intersubjetivas en la construcción social del conocimiento mediante lo que se puede denominar una subjetividad objetivante. Sin embargo, este proceso suscita la posibilidad de un acercamiento desde la imaginación, cuya característica es proporcionar insumos para la comprensión social del mundo.

Y un tercer apartado se embarca en la búsqueda de una relación entre un pensamiento simbólico, entendido laxamente, y a partir de todo lo expuesto anteriormente, como una primera impresión preracional de la realidad, y el conocimiento como una elaboración compleja que nace desde estas diversas dimensiones de lo humano en el mundo de la vida. 


\section{Conocimiento y ciencias sociales}

Una de las cualidades fundamentales de la especie humana radica en su capacidad cognoscitiva, es decir, los individuos tienen la necesidad de comprender el mundo en el que están situados, de manipular la materia o de construir sociedad. Así, el problema del conocimiento, en su expresión amplia, nos ha atado históricamente a debates filosóficos y científico-sociales sobre la construcción de la realidad, la verdad, la falsedad, lo objetivo o lo subjetivo. Por ello es pertinente generar un aporte y proporcionar una mirada más sobre este tema, en tanto se asume como un necesario ejercicio de reflexividad filosófico-sociológica.

En la lógica, como disciplina filosófica que estudia el pensamiento y sus estructuras, la ciencia sería en sí misma un sistema de conocimientos que se expresarían mediante métodos específicos. Esto ha sido abordado desde variadas disciplinas científico-sociales, como la antropología, la sociología o la psicología, donde cada una funcionaría como un sistema de conocimientos para construir conocimientos mediados por diversas variables socioculturales y aspectos sociocognitivos, actuando de forma cíclica en el tejido social y simbólico y otorgándonos ideas del mundo. Es decir, se van nutriendo constantemente de sí mismas a través de las relaciones entre diversos conocimientos emergentes. Por lo que el estudio del conocimiento ha conducido históricamente a la formación de agencias científico-sociales que intentan traspasar los problemas empíricos al pensamiento -y la racionalidad- a través de las ideas, por ejemplo la sociología del conocimiento, que en términos sintéticos podemos decir que "nace y se constituye alrededor del progresivo desvelamiento del carácter concreto y empírico del sujeto cognoscente" (Champourcin 1987:8) o bien una antropología del conocimiento, antes gnoseoantropología, que advierte a ese sujeto cognoscente como parte de una hermenéutica filosófica del lenguaje.

Estos elementos son importantes a la hora de comprender las inquietudes epistemológicas que suscita el proceso de investigación social, pues, en los estudios sociales de la ciencia, donde los conocimientos se clasifican por comunidades epistémicas, los diversos estilos de realizar y pensar el ejercicio científico manifestarían una indiscutible heterogeneidad, donde "se puede afirmar que la efectiva constitución de un campo científico responde a la articulación de un complejo conjunto de elementos que se van organizando en un espacio que se "autosustenta» y reproduce a través de las nuevas generaciones, es decir, a través de la conformación de nuevas tradiciones" (Vessuri y Canino 2007:88). Vale decir, el conocimiento, de alguna forma, se instituye imaginario-socialmente mediante las constantes reformulaciones teóricas que los elementos de la realidad y el investigador, como sujeto cognoscente, producen en relación en un mismo campo social. Pues, la ciencia social es tan tribal como cualquier otro grupo sociocultural, donde el conocimiento no está exento de la influencia pre-racional que lo sustenta, y donde "cada grupo o cultura tiene una Base Común (Common Ground) de conocimiento general, compartido e indiscutible, que es no-ideológico o pre-ideológico dentro de esa comunidad epistémica" (Van Dijk 2005:287). Esto impactará, o debe hacerlo, de alguna forma en el pensamiento científico y su propia identidad, por lo que considerar un desarrollo cognitivo en ciencias sociales como inmune a estímulos simbólicos e imaginarios, respecto de la realidad en la que se sustente, parece ser una idea que ha perdurado en el desarrollo del pensamiento social, pues: "Considerar el desarrollo cognitivo como la acumulación de competencias individuales en las cuales el orden sociocultural nada tiene que ver, es una idea que ha permeado tanto en las ciencias cognitivas (en su fase inicial) como en la filosofía de las ciencias y la epistemología sobre todo desde el punto de vista del dualismo cartesiano, en el que se fundamenta a su vez el método científico" (Fuentes 2017:256).

Las características multi-contextuales de las ciencias sociales hoy, entonces, plantean diversos mecanismos teóricos, epistemológicos y metodológicos para establecer las interrelaciones en sus campos 
de estudio, que a diferencia de las ciencias naturales basan su conocimiento en la relación sujeto-sujeto (la doble hermenéutica), donde se trataría: "de un mundo preinterpretado en el que los significados desarrollados por sujetos activos entran prácticamente en constitución o producción real de ese mundo" (Falleti 2006:72). Entonces, la diversidad y validez del conocimiento social radican en contemplar variadas dimensiones del proceso social y el contexto de acción que contiene en su núcleo todo un conjunto de conocimientos e ideas que sostienen explicaciones del mundo, de modo que cuando hablamos de conocimiento nos estamos refiriendo a todo un constructo humano, cognitivo y cultural, que es en sí mismo un complejo entramado de enunciados, representaciones teóricas y sociales del mundo que se manifiestan mediante la relación entre diversos actores del mundo social, pues, el conocimiento social emerge, en principio, debido a un acto hermenéutico-fenomenológico: el comprender e interpretar elementos abstractos, simbólicos, para hacerlos inteligibles $y$, posteriormente, validarlos e instituirlos mediante el conjunto de prácticas culturales que organizan la vida social: "La creación del consenso en torno a los significados de la realidad social es, pues, resultado de las interacciones de las que participan los sujetos en la vida cotidiana" (Rizo 2005:27). Asimismo, la función cognitiva, que funciona como matriz del conocimiento, conlleva una función simbólica, pues la realidad se interpreta desde símbolos que inundan la percepción de los individuos y donde el compuesto fundamental de aquellas experiencias de inteligibilidad proviene desde arquetipos que se materializan mediante una experiencia colectiva que será finalmente una apertura a la comprensión del mundo.

En las ciencias sociales el conocimiento se ha tratado de concebir como un constructo racional y donde la verdad en tanto tal se construye mediante "un relato tejido a partir de sugerencias y vislumbres que creemos nos ofrecen nuestros experimentos" (Bloor 2003:49). Aquí encontramos un problema que es necesario mencionar; la constante fricción entre verdad y creencia y la relación entre el conocimiento científico y el sentido común, donde la teoría del conocimiento construye jerarquizaciones mediante una suerte de niveles de conocimiento que marcan síntomas de prioridad y validez, a decir, el conocimiento científico, el teológico, el artístico y el conocimiento ordinario. Empero, podemos también concebir el conocimiento como un fenómeno total, en tanto que involucra las perspectivas científicas y sociales posibles; es socioantropológico y neurocognitivo a la vez, pues: "Si se acepta la acepción de la palabra "conocimiento» entonces la distinción entre la verdad y el error no es la misma que la distinción entre la experiencia individual (óptima) y la influencia social; se convierte, más bien, en una distinción dentro de la amalgama de experiencias y creencias socialmente mediadas que constituyen el contenido de una cultura" (Bloor 2003:49). Asimismo, para los científicos sociales el sentido común actúa como un depósito pre-teórico que actúa constantemente en la conciencia práctica y desde donde emergen los indicios que muchas veces direccionan las formas de construir conocimiento social, donde "el científico se expone siempre a hallar en las evidencias del sentido común residuos de teorías anteriores que la ciencia ya ha abandonado; dado que todo predispone a que los conceptos y teorías sociológicas pasen al dominio público, el sociólogo corre el riesgo, más que cualquier otro científico, de "retomar del fondo de conocimientos comunes, para volcarlos en la ciencia teórica»" (Bourdieu, Chamboredon y Passeron 2013:41). Por lo que, siguiendo la lógica de la sociología del conocimiento científico de estos autores, el proceso de ruptura para la sociología no necesariamente desplaza el conocimiento ordinario, o común, sino que lo somete a un rigor analítico fundamental y perpetuo.

De este modo, no podemos desdeñar la influencia del sentido común como una forma de conocimiento subjetivo, sobre las formulaciones teóricas que ascienden hacia una verdad desde las ciencias sociales, donde su premisa fundamental (la objetividad) se tiende a percibir como un absoluto en los mecanismos epistemológicos y metodológicos que se utilizan para enfrentar el descubrimiento social, sin que necesariamente se construya una convergencia entre lo objetivo y la subjetivo, o entre diversas formas de 
comprender el conocimiento, pero podemos entender que "La objetividad social (...) conforma tanto el factor subjetivo como las condiciones objetivas. La totalidad social no determina sólo el lado objetivo del proceso, sino también el factor subjetivo" (Guber 2004:56). Por lo que para la ciencia social: "La objetividad de la ciencia no podría descansar en un fundamento tan incierto como la objetividad de los científicos" (Bourdieu et. al 2013:106). De esta forma, es necesario siempre repensar el problema de la relación sujeto/objeto que, en tanto problema epistemológico, requiere una relación activa para comprender qué elementos influyen en la comprensión de la realidad social, y cómo esa realidad impregnada de sentidos diversos influye tanto en el desarrollo del conocimiento ordinario como en el proceso cognoscitivo científico social, puesto que el conocimiento siempre es producto de la estrecha relación entre el individuo y el mundo que le rodea, y es resultado de todo el cuerpo de significaciones que otorgan sentido a la existencia de los sujetos, donde los individuos han de experimentar socialmente la producción de sentidos en principio desde una dimensión cognitiva e imaginante, proveyendo respuestas comprensibles para el mundo social y donde "se requiere que ese mismo mundo significado sea, además, instituido o validado socialmente" (Baeza 2015:29).

Podemos desprender de esto que el conocimiento no se sitúa solo desde un proceso individual, como un natural producto de sí, sino que proviene de una cultura que "bajo esta perspectiva, es 'experiencia vivida', algo que denota un 'estilo de vida', históricamente contextualizado" (Gadea 2018:44). El conocimiento emerge desde los complejos procesos que vinculan al individuo con su entorno, con una cultura constituida por constantes interacciones simbólicas; es producto de su camino por un mundo de la vida que solo diferirá en estilos de conocer, pero que en tanto entramados de sentidos inciden "directamente en la forma en que las personas vivencian su entorno, definen la realidad y la verdad, perfilan el tipo de relación que mantienen con la naturaleza y con lo sobrenatural, erigen instituciones, simbolismos y rituales, orientan su acción cotidiana y se vinculan entre sí" (Toledo 2014:42).

\section{Subjetividad, objetividad e imaginación}

Para ir profundizando en el problema, podemos apreciar que la subjetividad se manifiesta, o se tiende a explicar habitualmente, como una suerte de experiencia íntima en los sujetos, en la cual este adquiere cualidades metafísicas e individualistas. Desde el idealismo, por ejemplo, los individuos adquieren significaciones del mundo mediante la relación entre las ideas, los objetos y la experiencia, donde una serie de signos y funciones cognitivas interactuantes en un mismo escenario social constituyen la materia prima para la construcción del conocimiento: "por lo tanto, lo subjetivo aparece mucho más como una referencia genérica para significar procesos del sujeto que conoce y construye, que como una definición ontológica particular de los fenómenos humanos" (González Rey 2008:227). Esta perspectiva nos abre la posibilidad de percibir una ontología de la comprensión a la manera de lo que Ricoeur llamó vía corta, donde la interrelación posible entre la cognoscencia y el sujeto-mundo constituyen el cambio epistemológico entre modos de conocimiento y modos de ser, marcando esa intersección fundamental entre la hermenéutica y la fenomenología. Por lo tanto: "Es necesario, entonces, salir deliberadamente del círculo encantado de la problemática del sujeto y del objeto, e interrogarse por el ser. Pero, para interrogarse por el ser en general, hay que interrogarse primero por ese ser que es el "ahí» de todo ser, el Dasein" (Ricoeur 2006:12). Vale decir, la comprensión (verstehen) no solo radica en formas de conocimiento mediante la experiencia con la exterioridad, sino que se constituye como parte de un individuo que tiene la capacidad de comprender y comprenderse como parte intrínseca de esa exterioridad en su tiempo y espacio social. Se conforma, si se quiere, como una totalidad. 
Esta perspectiva ontológica de la comprensión emerge como una agencia que busca de algún modo sustituir una epistemología de la interpretación, donde Husserl, por ejemplo, intentaría transformar la perspectiva del objeto como portador de intenciones mediante el campo de significaciones del sujeto, al igual que Heidegger, cuyas fenomenologías actúan críticas al objetivismo y de cierta manera se presentan como modos subjetivos de intención: "antes de la objetividad está el horizonte del mundo; antes del sujeto de la teoría del conocimiento está la vida operante, que algunas veces Husserl llama anónima, no porque vuelva por este rodeo a un sujeto impersonal kantiano, sino porque el mismo sujeto que tiene objetos se deriva de la vida operante" (Ricoeur 2006:14). Diremos, entonces, que la capacidad de conocimiento no radica tampoco desde una objetividad absoluta y cerrada en sí misma, sino que se enmarcaría en principio mediante un proceso de intersubjetividad.

El conocimiento científico positivista ha incorporado de alguna manera la idea de objetividad como principio canónico de la verdad, pero desde la filosofía antigua se ha considerado que el sujeto no es capaz de verdad en tanto no se transforme a sí mismo, un cambio en el modo de ser, evidente en la filosofía cartesiana, por ejemplo. Sin embargo, dirá Foucault que el sujeto no necesariamente debe transformarse a sí mismo, pues: "Basta con que sea lo que es para tener, en el conocimiento, un acceso a la verdad que está abierto para él por su estructura propia de sujeto" (Foucault 2002:190), por lo tanto, la idea de transformación es paradójica. De modo que el problema de la objetividad, entendemos, no puede tratarse sin el de la subjetividad, asumiendo esta como recipiente del pensamiento simbólico, puesto que cuando hablamos de la complejidad del conocimiento humano, implica el reconocimiento de un sujeto cognoscente que es también sujeto reflexivo (homo cogitans) y, por lo tanto, esa capacidad reflexiva actúa como principio germinal del acto de conocer mediante la relación sujeto/mundo. Entonces, la subjetividad "no es otra cosa que el modo en el cual se realiza la actividad cerebral de significación de los elementos de nuestro entorno (...) tenemos conciencia de algo cuando nuestra actividad cerebral se intenciona o se dirige hacia un algo particular (...) ella se prolonga "poblándose» con significaciones diversas, es decir, con características y atributos subjetivamente dados que configuran la apariencia de una totalidad significada" (Baeza 2015:32). Mientras que, por otro lado, la capacidad objetivante nos permite cosificar o racionalizar aquellos elementos que sustentan la vida social, por cuanto estos procesos cognitivos deben contener una serie de concatenaciones que dilucidan la situación del sujeto en el mundo, o bien, darle forma a esa totalidad sujeto/mundo.

Esta relación es compleja y, a favor de esta idea, las neurociencias han planteado algo sustancial; el trayecto precepto-concepto, o bien "la transformación de la información recogida por cualquiera de nuestros sentidos que luego es sometida a tratamiento en el cerebro" (Baeza 2015:31). Pues, si decimos que la objetividad es consecuencia de la subjetivación del mundo, debemos del mismo modo saber cómo se constituye un proceso intersubjetivo, donde la relación sujeto/mundo debe considerase como una relación entre lo simbólico, lo cognitivo y lo social, que en su función concomitante configuran mecanismos de comprensión del mundo y dirigen la experiencia social a través de las cualidades subjetivas primeras de todo individuo, o bien una cualidad ontológica que podemos denominar como simbólico-imaginativa que sustenta la experiencia humana a través de la construcción de referencias inteligibles de lo real, llegando a establecer incluso "una configuración imaginaria de nuevas realidades posibles" (RodríguezSalazar 2016:22). Es decir, la relación sujeto-objeto es en cierto modo una pista fundamental para entender la relación entre el pensamiento simbólico y el conocimiento, puesto que constituyen dos dimensiones imbricadas del individuo en el mundo de la vida y asimismo configuran un trayecto trascendental en el pensamiento científico social actual. 
De este modo, la imaginación, o la conciencia imaginante, no solo se afirma como un soporte del conocimiento social, ni tampoco manifiesta una oposición a la objetividad o el racionalismo, sino que conforma ese elemento abstracto fundamental en la capacidad de objetivar los procesos intersubjetivos del mundo, donde el conocimiento, finalmente, es también pensamiento simbólico. Lo diría Gilbert Durand cuando manifiesta que el conocimiento simbólico, en su desarraigo científico-social histórico, ha sido sepultado bajo la autoridad incorruptible de una episteme sujeta a paradigmas determinados ab initio mediante lo que denomina como ciencia iconoclasta: "frente a la imaginación comprensiva «que induce al error y la falsedad», la ciencia esgrimió las largas cadenas de razones de la explicación semiológica, asimilándolas en principio a las cadenas de la explicación positivista" (Durand 2007:25). De esta forma, se entiende que los individuos viven en una suerte de estructuras perpetuas de símbolos interpretables, lo que podemos comprender como arquetipos, que, en sentido amplio, constituyen la matriz del pensamiento simbólico mediante las influencias semánticas de todo contexto, promoviendo la intersubjetividad, pero que, no obstante, podríamos denominar el espíritu del conocimiento social.

Finalmente, todo se reduce a un problema epistemológico, pues la constante búsqueda de la verdad y la objetividad se fundamenta en conocer los principios que constituyen la esencia o naturaleza de algo, mientras que los medios que esta búsqueda requiera, estrictamente relacionados al método, han de poner en cuestión los mecanismos sociocognitivos, la imaginación, la significación y todo el conjunto de elementos que inciden en el problema que emerge desde la complejidad cognoscitiva en las ciencias sociales y en la vida social misma.

\section{Conocimiento y pensamiento simbólico}

Problematizar el conocimiento desde esta perspectiva, entonces, es comprenderlo como resultado de la interiorización de un mundo simbolizado y en principio subjetivado, es esa la fortaleza del pensamiento simbólico. Donde la realidad es concebida desde un acto cognitivo en función de redes simbólicas, sentidos y prácticas socioculturales que marcan un autenticismo desde las cualidades únicas que presentan los diversos marcos de referencia simbólico-sociales que organizan la vida social, en su diversidad históricocultural, $y$, por lo tanto, todo el proceso intersubjetivo que vincula a los individuos con el mundo no puede simplemente desprenderse de la realidad concreta, puesto que: "Una situación, entendida también como una manera de organización de la experiencia, se relaciona no únicamente con un individuo que aisladamente otorga significados a un acontecimiento, sino también con un repertorio cultural" (Gadea 2018:51). Esto marca una diferencia con aquella perspectiva realista que considera la relación entre el individuo y un mundo exterior a él, el cual debe conocer, asumimos acá una interrelación.

Esto no es algo que simplemente pueda desdeñarse en esa búsqueda racional de la experiencia humana, pues, debemos comprender que todo sujeto es a la vez homo symbolicus y este expresará en sí mismo una totalidad: "The name Homo symbolicus describes us well; almost everything we do has symbolic undertones. It is therefore not surprising that we expect people like us in the past to have depended equally on symbolism, which would have demanded the use of some form of language" (Wadley 2011:97). Desde este punto de vista, los individuos deben interpretar aquellos signos que hacen inteligible esas relaciones, encontrándose el sujeto en una inextricable relación simbólica con todo lo que le rodea. Asimismo, esta función simbólica conduce a un conjunto de significaciones sociales que actúan en el entramado social como una suerte de magma; instituciones o conjunto de significaciones validadas socialmente, donde la influencia interpretativa está marcada por estas que, aunque extensas en complejidad, son simbólicamente insoslayables: "Las instituciones no se reducen a lo simbólico, pero no 
pueden existir más que en lo simbólico" (Castoriadis 2007:187), proporcionando los fundamentos de toda sociedad.

Más allá, la objetivación del conocimiento, para la ciencia social, constituye un elemento que funcionará como soporte teorizado de la realidad, pues: "El componente teórico del conocimiento es un componente social, y es una parte necesaria de la verdad, no un signo de un mero error" (Bloor 2003:50). Por lo que los imaginarios sociales, en tanto redes simbólicas, no existen independiente de las instituciones sociales que promueven la emergencia del conocimiento social, $y$, en consecuencia, adquirieren fuerza mediante las decisiones de los sujetos al tomar opciones de vida. Lo imaginario en este punto se manifiesta como una relación recíproca entre una red de relaciones simbólicas, discursivas y prácticas sociales, instalándose en estas instituciones que configuran socialmente la realidad y que la explican a través de tendencias valorativas que los individuos expresan en el juzgar y actuar. Lo imaginario: "Se instala en las distintas instituciones que componen la sociedad, para poder actuar en todas las instancias sociales" (Díaz 1996:13).

Entonces, la ciencia social se constituiría como un subconjunto del conjunto de todos los conocimientos del mundo social, donde se tiende a concebir una proposición sujeta a lo verdadero y lo falso, lo que constituye el problema de asumir una dicotomía entre un conocimiento "verdadero-objetivo" y otro "falso-subjetivo", por lo que es necesario establecer una autocrítica constante, si se quiere, una vigilancia epistemológica sobre la forma en que asumimos, en tanto que investigadores e investigadoras, la comprensión y construcción del conocimiento. Pues, su importancia radica en la capacidad de los investigadores para construir referencias teóricas que den cuenta de la relación entre los individuos y el mundo, y que desde un comienzo se encuentra influenciado por un marco de referencia simbólica que resulta, o culmina, en un revoltijo entre experiencias, nociones y creencias compartidas. No hay que caer en un falso distanciamiento para establecer un principio de objetividad en el quehacer científico social, sino que es necesario asumir de entrada que, en lo que incumbe a los estudios sociales, el científico siempre hace parte del contexto que interpreta, pues: "la experiencia de conocimiento es aquella que se logra en condiciones que permiten a un sujeto descubrirse en las conexiones de su realidad, pasando por un trance en el que se ve expuesto a comprenderlas bajo la dirección sabia de otros" (Madrid 2011:14).

Así, la verdad en las ciencias sociales no es otra cosa que una visión teórica del mundo en un tiempo y espacio determinado: "el conocimiento propio de nuestra cultura, tal y como se representa en nuestra ciencia, no es el conocimiento de una realidad que cualquier individuo pueda experimentar o aprender por sí mismos, sino lo que nuestras teorías mejor contrastadas y nuestros pensamientos más elaborados nos dicen, pese a lo que puedan decir las apariencias" (Bloor 2003:49). Si comprendemos la relación intrínseca entre el conocimiento, lo simbólico y la experiencia, debemos tener consciencia de las reformulaciones posibles respecto a la propia comprensión de la existencia, apartándonos de los márgenes establecidos como experiencia práctica y situándolo también en las formas que impulsan la comprensión social mediante el pensamiento simbólico, uno que generalmente ha sido atribuido a cualidades psicoanalíticas sobre la función del inconsciente, y que ha sido centro de atracción para la psicología, la antropología o la sociología. El punto es la importancia de considerar la compleja totalidad que expresa el problema del conocimiento, donde: "es importante considerar que muchos de los estudios clásicos que se ocuparon del problema de la construcción del conocimiento, estuvieron orientados por un enfoque metateórico que disociaba los componentes de la experiencia social de los sujetos, centrándose en uno de ellos o reduciendo unos a otros" (García-Palacios, Horn y Castorina 2015:867). Por lo que la complejidad analítica en el problema del conocimiento y su relación con lo simbólico implica una necesaria relación entre diversas perspectivas en función -necesaria- de construir un diálogo enraizado a una filosofía que inunde las posibilidades sociológicas (sensu lato) para darle forma a un cuerpo teórico-epistemológico que 
aborde el problema del conocimiento desde un nuevo espíritu científico, uno que no tema realizar un tratado transdisciplinario para visualizar una perspectiva más holística del conocimiento humano, científico y social y su inextricable relación.

\section{Consideraciones finales}

El proceso de construcción de conocimiento en ciencias sociales debe su anatomía a diversos cambios en su propia analítica, cuyos fundamentos varían históricamente, especialmente desde el positivismo a la hermenéutica y la fenomenología como bases epistémicas que sirven para advertir la perspectiva crítica de este artículo. Así, una triangulación entre el conocimiento y el pensamiento simbólico, en tanto categorías analíticas, no tiene otro fundamento que visualizar la intersubjetividad, lo simbólico y cognitivo como actuantes activos en las formas de ver, comprender y apropiarse del mundo social, como también de reproducirlo empírica y teóricamente desde una perspectiva del sentido común hasta el conocimiento científico social. Por lo tanto, para poder comprender de forma sintética esta relación se ha establecido una serie de elementos que constituirían esa totalidad, aportando una mirada particular sobre el problema del conocimiento.

El conocimiento emerge desde el mundo social a través de las posibilidades que la estructura cognitiva provee en relación con la experiencia y los elementos simbólicos e institucionales que imperativamente inducen los procesos de significación. Para ello, hemos establecido lo que denominamos una tríada fundamental que se nutre por la imaginación, la subjetividad y la objetividad, cuyas características no deben comprenderse distantes entre sí, sino que actúan en interrelación, por cuanto la objetividad emerge tras un proceso de intersubjetividad social que basa sus posibilidades por la capacidad reflexiva y simbólica de los individuos. Pero estos elementos fundamentales se complejizarán al intentar encontrar respuestas al problema del conocimiento mediante estas tres dimensiones interrelacionadas: a) la base que constituye la cualidad imaginativa de los individuos y le otorga las facultades cognoscitivas primeras; b) la subjetividad como el primer proceso de vinculación comprensiva entre los sujetos y el mundo, y c) la objetividad que asume la convalidación intersubjetiva como la posibilidad de otorgar un sentido compartido de la realidad a los individuos. Donde, finalmente, estos elementos funcionarían como parte de su estructura cognoscitiva y, asimismo, nos adentra en la problematización del nexo entre el conocimiento y el pensamiento simbólico como elementos que deben pensarse desde una perspectiva socioantropológica (holística) para comprender que esta relación nos posibilita el acceso a los principios fundamentales en la construcción de la realidad social a través del acto de conocer.

En definitiva, esta perspectiva nos permite formular ideas acerca de los elementos que en conjunto e interrelación emergen como parte del sustrato del conocimiento, nos hacen percibir que el pensamiento humano y el conocimiento científico y social siempre se ven influenciados por estructuras simbólicas y conjuntos de significaciones, y donde la experiencia se convierte en un principio fundamental de la realidad y su explicación. Es interesante concebir el proceso cognoscitivo de lo humano desde una perspectiva sociofenomenológica, donde asumimos la premisa que "la sociedad es un conjunto de personas que actúan en el mundo y cuyas acciones tienen sentido; y es relevante tratar de comprender este sentido para poder explicar los resultados del accionar de los sujetos" (Rizo 2005:24). Estos elementos, además, nos generan cuestionamientos acerca de una conexión fundamental, sino la más fundamental de todas; la relación entre el conocimiento y lo real, puesto que problematizar el pensamiento simbólico y el conocimiento promueve, imperativamente, la reflexión respecto de los elementos que podemos considerar en nuestras formulaciones investigativas, cuya sustancia emerge desde un mundo empírico, a la vez que simbólico e imaginario. 


\section{Bibliografía}

Baeza, M. 2015. Hacer mundo. Significaciones imaginario-sociales para constituir sociedad. Santiago: RIL editores.

Bloor, D. 2003. Conocimiento e imaginario social. Barcelona: Gedisa.

Bourdieu, P ; Chamboredon, J.C ; Passeron, J.C. 2013. El oficio de sociólogo: presupuestos epistemológicos. Madrid: Siglo XXI.

Brunetti, J. 2013. Thomas Kuhn: ¿epistemólogo o psicólogo de la ciencia? Revista iberoamericana de ciencia tecnología y sociedad 8(22): 191-212. https://dialnet.unirioja.es/servlet/articulo?codigo $=4227125$

Castoriadis, C. 2007. La institución imaginaria de la sociedad. Buenos Aires: Tusquets Editores.

Champourcin, E. 1987. El estatuto teórico de la sociología del conocimiento. Reis: Revista española de investigaciones sociológicas 40: 7-44. https://dialnet.unirioja.es/servlet/articulo?codigo=249164

Díaz, E. 1996. La ciencia y el imaginario social. Madrid: Biblos.

Durand, G. 2007. La imaginación simbólica. Buenos Aires: Amorrortu.

Falleti, V. 2006. Los problemas de la construcción del conocimiento en las ciencias sociales. Universitas Humanística 62(62): 71-89.

http://revistas.javeriana.edu.co/index.php/univhumanistica/article/view/2205

Fuentes, F. 2017. El cambio de conceptos y teorías en el conocimiento científico y ordinario. Revista Educación y Humanismo 19(33): 253-270. http://dx.doi.org/10.17081/eduhum.19.33.2643

Foucault, M. 2002. La hermenéutica del sujeto: curso en el Collège de France, 1981-1982. Buenos Aires: Fondo de Cultura Económica.

Gadea, C. 2018. El interaccionismo simbólico y sus vínculos con los estudios sobre cultura y poder en la contemporaneidad. Sociológica 33(95): 39-64. http://www.sociologicamexico.azc.uam.mx/index.php/Sociologica/article/view/1392

García-Palacios, M; Horn, A; Castorina, J. 2015. El proceso de investigación de conocimientos infantiles en psicología genética y antropología. Revista Latinoamericana de Ciencias sociales, Niñez y Juventud 13(2): 865-877. https://doi.org/10.11600/1692715x.13222090215

González Rey, F. 2008. Subjetividad social, sujeto y representaciones sociales. Diversitas 4(2): 225-243. http://pepsic.bvsalud.org/scielo.php?script=sci abstract\&pid=S1794-99982008000200002

Guber, R. 2004. El salvaje metropolitano: reconstrucción del conocimiento social en el trabajo de campo. Buenos Aires: Paidós.

Madrid, M. 2011. El conocimiento social y el compromiso del investigador en el mundo contemporáneo. Intersticios Sociales 1(1): 1-19. http://www.redalyc.org/articulo.oa?id=421739489002

Narváez, V. 2014. El concepto de ciencia como sistema, el positivismo, neopositivismo y las "investigaciones cuantitativas y cualitativas". Salud Uninorte 30(2): 227-244. http://rcientificas.uninorte.edu.co/index.php/salud/article/viewFile/5490/7003

Kuhn, T. 1971. La estructura de las revoluciones científicas. México: Fondo de Cultura Económica 
Ricoeur, P. 2006. El conflicto de las interpretaciones: ensayos de hermenéutica. Buenos Aires: Fondo de Cultura Económica.

Rizo, M. 2005. La psicología social y la sociología fenomenológica. Global Media Journal México 2(3): 19-33. https://gmjei-ojs-tamiu.tdl.org/gmjei/index.php/GMJ El/article/view/128/125

Rodríguez-Salazar, L. 2016. El cambio teórico en ciencia desde una epistemología de la imaginación. Elementos 101: 21-27. http://elementos.buap.mx/num101/htm/21.htm

Toledo, U. 2014. El programa sociofenomenológico de investigación, pp. 39-67. En: F. Osorio. Epistemología y ciencias sociales: ensayos latinoamericanos. Santiago: LOM ediciones.

Thompson, M. 2005. Phenomenology of intersubjectivity: a historical overview of the concept and its clinical implications, pp. 35-70. In: J. Mills. Relational and intersubjective perspectives in psychoanalysis. Maryland: Rowman and Littlefield Publishers.

Van Dijk, T. 2005. Discurso, conocimiento e ideología. Reformulación de viejas cuestiones y propuesta de algunas soluciones nuevas. Cuadernos de Información y Comunicación 10: 285-318. https://revistas.ucm.es/index.php/CIYC/article/view/CIYC0505110285A

Vessuri, H. y Canino, M. 2007. Los estudios sociales de la ciencia y la tecnología. Revista Venezolana de Economía y Ciencias Sociales 13(1): 87-90. http://www.scielo.org.ve/scielo.php?script=sci arttext\&pid=S1315-64112007000100005

Vidal, R. 2011. El giro epistemológico hermenéutico en la última tradición científica moderna. Cinta de moebio 40: 22-46. https://doi.org/10.4067/S0717-554X2011000100002

Wadley, L. 2011. Complex cognition required for compound adhesive manufacture in the Middle Stone Age implies symbolic capacity, pp 97-110. In: C. Henshilwood \& F. D'Errico. Homo symbolicus: the dawn of language, imagination and spirituality. Amsterdam: John Benjamins.

Recibido el 11 Abr 2019

Aceptado el 16 Jun 2019 